\title{
Model Reference Tracking Control for Nonlinear Systems described by Takagi-Sugeno Structure
}

\author{
(Invited Paper: SS-19 Recent Advances in Fuzzy Model-Based Control Design and Analysis)
}

\author{
Souad Bezzaoucha*†, Benoît Marx* ${ }^{* \dagger}$, Didier Maquin*†, José Ragot*† \\ *Université de Lorraine, CRAN, UMR 7039, 2 avenue de la Forêt de Haye, Vandoeuvre-lès-Nancy Cedex, 54516, France \\ †CNRS, CRAN, UMR 7039, France. \\ e-mail:firstname.name@univ-lorraine.fr.
}

\begin{abstract}
This work concerns the model reference tracking control problem for nonlinear systems represented by TakagiSugeno (T-S) models, with a guaranteed $\mathcal{L}_{2}$ performance to attenuate the tracking error for bounded reference inputs. The objective is to make the system state follow as closely as possible the model reference state. The control scheme is based on a parallel distributed compensation controller fed by a state observer for Takagi-Sugeno systems with unmeasurable premise variables. The observer/controller synthesis is formulated in terms of linear matrix inequalities. Systems with noise output measurements are also envisaged with the use of filter. Simulation example are given to illustrate the design procedures and tracking performance of the proposed approach.
\end{abstract}

Keywords-Takagi-Sugeno systems, observer-based control, reference tracking

\section{INTRODUCTION}

In the control theory, the tracking control design is an important subject especially for its practical applications. For nonlinear systems design, numerous methods were introduced, like the exact feedback linearization, the sliding mode and the adaptive control.

The feedback linearization technique has been introduced to deal with nonlinear systems [1]. However, because of its complicated control algorithm, the fact that the stability of the controller is not guaranteed for nonminimum phase systems and its application to complex nonlinear systems is tedious. The sliding mode control presents the advantage of the robustness to uncertainties [2] but is too sensitive to the chattering phenomenon.

An interesting and efficient way to estimate and control complex nonlinear systems is to write them in a simpler form, like the Takagi-Sugeno (T-S) model. Originally introduced by [3], the T-S representation allows to exactly describe nonlinear systems, under the condition that the nonlinearities are bounded. This is reasonable since state variables as well as parameters of physical systems are bounded, see for example [4], [5], [6] and the references therein.

Despite an abundant literature on stability conditions of $\mathrm{T}$ $\mathrm{S}$ models, few authors have dealt with the tracking problem recently. Some works are concerned with state feedback, $H \infty$ performances [7], [8] and output feedback [9]. Mostly available results for Parallel Distributed Compensation (PDC) structure are formuled as Bilinear Matrix Inequalities (BMI) and two step algorithm is generally used [10].

In the present work, the nonlinear tracking control problem is expressed in terms of Linear Matrix Inequality (LMI). Based on the T-S and PDC structures, with a suitable choice of the reference model, a $\mathcal{L}_{2}$ tracking performance related to tracking error is formulated. Then a T-S observer-based controller is developed to minimize the tracking error. The contribution of the proposed approach compared to the previous works, is the statement of an LMI formulation for the problem of trajectory tracking for nonlinear systems represented by T-S models, with $\mathcal{L}_{2}$ performance and state feedback control. The adopted strategy is based on a descriptor approach allowing to reduce the computational cost by avoiding the crossing terms between the feedback gains and the Lyapunov matrices in the closed-loop dynamics.

The synthetized controller is based on a state observer. It is also important to highlight that the unmeasurable premise variables case is considered here, whereas in most of the previous works [9], [10] the premise variables as supposed to be available.

The paper is presented as follows: in section II, the tracking criteria as well as the observer design and the tracking control law are introduced. In section III, in order to minimize the tracking error using an exact estimation of the system state, a static PDC observed state feedback tracking control is formulated in terms of LMI. In section IV a numerical example is presented to illustrate the effectiveness of the proposed approach. In section $\mathrm{V}$, the influence of an output measurement noise is taken into account and minimized when designing the observer and controller. A numerical example and some simulation results are given in section VI. Conclusions are exposed in section VII.

\section{PROBLEM STATEMENT}

\section{A. Tracking criteria}

In order to design a model reference tracking control law for nonlinear systems, a T-S representation is considered. The T-S modeling allows the representation of the behavior of nonlinear systems by the interpolation of a set of linear submodels. Each submodel contributes to the global behavior of the nonlinear system through a weighting function $\mu_{i}(\xi(t))$ [6]. The T-S structure is given by

$$
\left\{\begin{aligned}
\dot{x}(t) & =\sum_{i=1}^{n} \mu_{i}(\xi(t))\left(A_{i} x(t)+B_{i} u(t)\right) \\
y(t) & =C x(t)
\end{aligned}\right.
$$

where $x(t) \in \mathbb{R}^{n_{x}}$ is the system state, $u(t) \in \mathbb{R}^{n_{u}}$ the control input and $y(t) \in \mathbb{R}^{m}$ the system output. In this paper, a focus is made on the T-S with premise variables $\xi(t) \in \mathbb{R}^{q}$ depending 
on the state variable $x(t)$ and thus being unmeasurable. In this case, let us denote $\mu_{i}(\xi(t))=h_{i}(x(t))$. These weighting functions satisfy the so-called convex sum property

$$
\left\{\begin{array}{l}
\sum_{i=1}^{n} h_{i}(x(t))=1 \\
0 \leq h_{i}(x(t)) \leq 1, \quad i=1, \ldots, n
\end{array}\right.
$$

The following stable linear reference model is considered [10]:

$$
\dot{x}_{r}(t)=A_{r} x_{r}(t)+B_{r} u_{r}(t)
$$

where $x_{r}(t)$ is the reference state to be followed by $x(t)$ and $u_{r}(t)$ is the bounded reference input. The matrices $A_{r}$ and $B_{r}$ are designed according to the desired performances of the time response of the reference model.

The tracking error is defined by:

$$
e_{r}(t)=x(t)-x_{r}(t)
$$

Then the objective is to design a PDC controller, stabilizing the nonlinear system (1) and ensuring the following $\mathcal{L}_{2}$ tracking performance [10], [11], [12]:

$$
\int_{0}^{t_{f}} e_{r}^{T}(t) Q e_{r}(t) d t \leq \eta^{2} \int_{0}^{t_{f}} u_{r}^{T}(t) u_{r}(t) d t
$$

where $Q$ is a positive definite weighting matrix and $\eta$ the prescribed attenuation level. The matrix $Q$ is chosen accordingly to the state components for which some specific following is desired.

In (5), the upper bound of the $\mathcal{L}_{2}$ gain from $u_{r}(t)$ to $e_{r}(t)$ denoted by $\eta$ quantifies the effect of the reference input on the weighted tracking error. Obviously, the objective is to minimize $\eta$.

\section{B. Observer design}

In the present work, the case where the state variables are not available for the feedback control with unmeasurable premise variable is considered. Therefore, the following T-S observer is considered to deal with the state estimation of T-S nonlinear system (1):

$$
\left\{\begin{array}{l}
\dot{\hat{x}}(t)=\sum_{i=1}^{n} h_{i}(\hat{x}(t))\left(A_{i} \hat{x}(t)+B_{i} u(t)+L_{i}(y(t)-\hat{y}(t))\right) \\
\hat{y}(t)=C \hat{x}(t)
\end{array}\right.
$$

where $\hat{x}(t) \in \mathbb{R}^{n_{x}}$ is the estimated system state and $L_{i} \in$ $\mathbb{R}^{n_{x} \times m}$ are the observer gains.

The reader can note that the selected observer (6) is a classical proportional one. A more general observer like a proportional integral or a multi-integral may also be used.

Let us define the state estimation error $e_{x}(t)$ as

$$
e_{x}(t)=x(t)-\hat{x}(t)
$$

Its dynamics cannot be easily computed directly from (7) since in equation (1) the weighting functions depend on the unmeasurable variable $x(t)$ whereas those of (6) depend on its estimate $\hat{x}(t)$. Because of that, based on the convex sum property of the weighting functions, the state equation (1) need to be rewritten as follow [13]

$$
\begin{aligned}
\dot{x}(t)=\sum_{i=1}^{n}\left[h_{i}(\hat{x}(t))\left(A_{i} x(t)+B_{i} u(t)\right)+\right. \\
\quad\left(h_{i}(x(t))-h_{i}(\hat{x}(t))\left(A_{i} x(t)+B_{i} u(t)\right)\right]
\end{aligned}
$$

This form allows a better comparison of $x(t)$ with $\hat{x}(t)$, since $h_{i}(\hat{x}(t))$ not only appears in (6), but also in (8). Let us define:

$$
\begin{aligned}
\Delta A(t) & =\sum_{i=1}^{n}\left[h_{i}(x(t))-h_{i}(\hat{x}(t)] A_{i}\right. \\
& =\mathcal{A} \Sigma_{A}(t) E_{A}
\end{aligned}
$$

and

$$
\begin{aligned}
\Delta B(t) & =\sum_{i=1}^{n}\left[h_{i}(x(t))-h_{i}(\hat{x}(t)] B_{i}\right. \\
& =\mathcal{B} \Sigma_{B}(t) E_{B}
\end{aligned}
$$

with

$$
\begin{aligned}
& \mathcal{A}=\left[\begin{array}{lll}
A_{1} & \ldots & A_{n}
\end{array}\right], \Sigma_{A}(t)=\operatorname{diag}\left(\delta_{1}(t) I_{n_{x}}, \ldots, \delta_{n}(t) I_{n_{x}}\right), \\
& \mathcal{B}=\left[\begin{array}{lll}
B_{1} & \ldots & B_{n}
\end{array}\right], \Sigma_{B}(t)=\operatorname{diag}\left(\delta_{1}(t) I_{n_{u}}, \ldots, \delta_{n}(t) I_{n_{u}}\right), \\
& E_{A}=\left[\begin{array}{lll}
I_{n_{x}} & \ldots & I_{n_{x}}
\end{array}\right]^{T}, E_{B}=\left[\begin{array}{lll}
I_{n_{u}} & \ldots & I_{n_{u}}
\end{array}\right]^{T} \\
& \delta_{i}(t)=h_{i}(x(t))-h_{i}(\hat{x}(t))
\end{aligned}
$$

where $\operatorname{diag}\left(A_{1}, \ldots, A_{n}\right)$ refers to a block diagonal matrix with the square matrices $A_{1}, \ldots, A_{n}$ on its diagonal.

From (2) and (11), it follows:

$$
-1 \leq \delta_{i}(t) \leq 1
$$

implying

$$
\Sigma_{A}^{T}(t) \Sigma_{A}(t) \leq I, \quad \Sigma_{B}^{T}(t) \Sigma_{B}(t) \leq I
$$

Using (9) and (10), the system (8) is then written as an uncertain system given by:

$$
\left.\dot{x}(t)=\sum_{i=1}^{n} h_{i}(\hat{x}(t))\left(A_{i}+\Delta A(t)\right) x(t)+\left(B_{i}+\Delta B(t)\right) u(t)\right)
$$

From equations (14), (7) and (6), the dynamics of the state estimation error is given by

$$
\begin{aligned}
& \dot{e}_{x}(t)=\sum_{i=1}^{n} h_{i}(\hat{x}(t))\left(\left(A_{i}-L_{i} C\right) e_{x}(t)\right. \\
& +\Delta A(t) x(t)+\Delta B(t) u(t))
\end{aligned}
$$

\section{Tracking control}

The control structure is choosen as a PDC law [14], sharing the same weighting functions as those of the system observer:

$$
u(t)=-\sum_{i=1}^{n} h_{i}(\hat{x}(t)) K_{i}\left(x_{r}(t)-\hat{x}(t)\right)
$$

where $K_{i} \in \mathbb{R}^{n_{u} \times n_{x}}$ are the controller gains.

The study objective is to compute the controller gains $K_{i}$ as well as the observer gains $L_{i}$ ensuring the asymptotic stability of the closed-loop model (18)-(see the next section) and guaranteeing the $\mathcal{L}_{2}$ tracking performance (5). 


\section{T-S STATE FEEDBACK TRACKING CONTROL DESIGN}

The aim of the control input (16) in system equation (1) is to make the system state $x(t)$ track the reference state $x_{r}(t)$ (3) as closely as possible.

To achieve this purpose, the descriptor approach is considered. Based on redundancy property, the closed loop system is represented as an implicit or singular system [15], [16]. This approach is well known to relax and reduce the computational cost of LMI conditions by avoiding the appearance of crossing terms between the feedback gains and the Lyapunov matrices in the closed loop dynamics. As a consequence, the number of LMI decreases and relaxed conditions are obtained [17], [18], [19].

The control law (16) is then written as follows

$$
0 . \dot{u}(t)=-\sum_{j=1}^{n} h_{i}(\hat{x}(t)) K_{i}\left(x_{r}(t)-\hat{x}(t)\right)-u(t)
$$

From definitions (4), (15), (3) and (17), considering the augmented vector $x_{a}(t)=\left(\begin{array}{llll}e_{r}^{T}(t) & e_{x}^{T}(t) & x_{r}^{T}(t) & u^{T}(t)\end{array}\right)^{T}$, its dynamic may be written as the following:

$$
E \dot{x}_{a}(t)=\sum_{i=1}^{n} h_{i}(\hat{x}(t))\left(\bar{A}_{i}(t) x_{a}(t)+\bar{B} u_{r}(t)\right)
$$

with

$$
\begin{aligned}
& \bar{A}_{i}(t)=\left(\begin{array}{cccc}
A_{i}+\Delta A(t) & 0 & A_{i}-A_{r}+\Delta A(t) B_{i}+\Delta B(t) \\
\Delta A(t) & A_{i}-L_{i} C & \Delta A(t) & \Delta B(t) \\
0 & 0 & A_{r} & 0 \\
K_{i} & -K_{i} & 0 & -I_{n_{u}}
\end{array}\right) \\
& E=\left(\begin{array}{cccc}
I_{n_{x}} & 0 & 0 & 0 \\
0 & I_{n_{x}} & 0 & 0 \\
0 & 0 & I_{n_{x}} & 0 \\
0 & 0 & 0 & 0
\end{array}\right), \bar{B}=\left(\begin{array}{c}
-B_{r} \\
0 \\
B_{r} \\
0
\end{array}\right)
\end{aligned}
$$

Note that with the augmented vector $x_{a}(t)$, the tracking criteria (5) becomes:

$$
\int_{0}^{t_{f}} x_{a}^{T}(t) Q_{a} x_{a}(t) d t \leq \eta^{2} \int_{0}^{t_{f}} u_{r}^{T}(t) u_{r}(t) d t
$$

with $Q_{a}=\operatorname{diag}(Q, 0,0,0)$. The solution to the tracking problem (computation of the gains $K_{i}$ and $L_{i}$ ) is given by the following theorem:

Theorem 1. There exist a PDC state feedback controller (16) and an observer (6) for a nonlinear system described by a T-S model (1) guaranteeing the $\mathcal{L}_{2}$ tracking performance (5) for a prescribed value $\eta^{2}$, if there exists matrices $P_{1}=P_{1}^{T}>0$, $P_{2}=P_{2}^{T}>0, P_{3}=P_{3}^{T}>0, P_{4}>0, F_{i}, R_{i}$, positive scalars $\lambda_{1}^{1}, \lambda_{3}^{1}, \lambda_{5}^{1}, \lambda_{1}^{2}, \lambda_{3}^{2}$ and $\lambda_{4}^{2}$ satisfying the LMI (21) for $i=1, \ldots, n$, with

$$
\begin{aligned}
& M_{i}^{1}=A_{i}^{T} P_{1}+P_{1} A_{i}+Q+\left(\lambda_{1}^{1}+\lambda_{1}^{2}\right) E_{A}^{T} E_{A} \\
& M_{i}^{2}=P_{2} A_{i}+A_{i}^{T} P_{2}-C^{T} F_{i}^{T}-F_{i} C \\
& M^{3}=A_{r}^{T} P_{3}+P_{3} A_{r}+\left(\lambda_{3}^{1}+\lambda_{3}^{2}\right) E_{A}^{T} E_{A} \\
& M^{4}=-P_{4}-P_{4}^{T}+\left(\lambda_{4}^{1}+\lambda_{4}^{2}\right) E_{B}^{T} E_{B}
\end{aligned}
$$

The controller and observer gains are given $(i=1, \ldots, n)$ by

$$
\begin{aligned}
& K_{i}=\left(P_{4}^{T}\right)^{-1} R_{i} \\
& L_{i}=P_{2}^{-1} F_{i}
\end{aligned}
$$

Remark 1. The proposed tracking problem solution is based on an $\mathcal{L}_{2}$ attenuation with a prescribed value $\eta^{2}$. One can also ensure the tracking with the smallest admissible attenuation by solving the optimization problem (23)

$$
\left\{P_{1}, P_{2}, P_{3}, P_{4}, F_{i}, R_{i}, \lambda_{1}^{1}, \lambda_{3}^{1}, \lambda_{5}^{1}, \lambda_{1}^{2}, \lambda_{3}^{2}, \lambda_{4}^{2}\right\}^{\bar{\eta}}
$$

such that condition (21) is guaranteed with $\bar{\eta}=\eta^{2}$.

Proof:

Let us consider the following Lyapunov function

$$
V(t)=x_{a}^{T}(t) E^{T} P x_{a}(t)
$$

with the condition

$$
E^{T} P=P^{T} E \geq 0
$$

From (25) and (19), the matrix $\mathrm{P}$ is chosen as a block diagonal matrix $P=\operatorname{diag}\left(P_{1}, P_{2}, P_{3}, P_{4}\right)$ with

$$
P_{1}=P_{1}^{T}>0, P_{2}=P_{2}^{T}>0, P_{3}=P_{3}^{T}>0
$$

Using (18), the time derivative of $V(t)$ is the following:

$$
\begin{aligned}
\dot{V}(t)= & \dot{x}_{a}^{T}(t) E^{T} P x_{a}(t)+x_{a}^{T}(t) P^{T} E \dot{x}_{a}(t) \\
= & \sum_{i=1}^{n} h_{i}(\hat{x}(t))\left(x_{a}^{T}(t)\left(\bar{A}_{i}^{T}(t) P+P^{T} \bar{A}_{i}(t)\right) x_{a}(t)\right. \\
& \left.+x_{a}^{T}(t) P^{T} \bar{B} u_{r}(t)+u_{r}^{T}(t) \bar{B}^{T} P x_{a}(t)\right)
\end{aligned}
$$

To ensure the stability of the closed loop system (18) and the $\mathcal{L}_{2}$ attenuation (20), with the Lyapunov function (24), the condition to verify is:

$$
\dot{V}(t)+x_{a}^{T}(t) Q_{a} x_{a}(t)-\eta^{2} u_{r}^{T}(t) u_{r}(t)<0
$$

From equations (26), (19) and (20), (27) is written as:

$$
\begin{aligned}
& \sum_{i=1}^{n} h_{i}(\hat{x}(t))\left(\begin{array}{ll}
x_{a}^{T}(t) & \left.u_{r}^{T}(t)\right)
\end{array}\right. \\
& \left(\begin{array}{cc}
\bar{A}_{i}^{T}(t) P+P^{T} \bar{A}_{i}(t)+Q_{a} & P^{T} \bar{B} \\
\bar{B}^{T} P & -\eta^{2} I
\end{array}\right)\left(\begin{array}{l}
x_{a}(t) \\
u_{r}(t)
\end{array}\right)<0
\end{aligned}
$$

which is satisfied if:

$$
\left(\begin{array}{cc}
\bar{A}_{i}^{T}(t) P+P^{T} \bar{A}_{i}(t)+Q_{a} & P^{T} \bar{B} \\
\bar{B}^{T} P & -\eta^{2} I
\end{array}\right)<0
$$

In order to solve the matrix inequality above, a solution consists into decomposing the matrix into two terms: the constant term and the time dependent one. Then, considering the definitions (9) and (10) with property (13), the time dependent term will be bounded.

Using definition (19), the BMI to solve is then

$$
\overline{\mathcal{A}_{i}}+Q(t)+Q^{T}(t)<0
$$

with $\overline{\mathcal{A}_{i}}$ and $Q(t)$ defined as:

$\overline{\mathcal{A}_{i}}=\left(\begin{array}{ccccc}\bar{M}_{i}^{1} & 0 & P_{1}\left(A_{i}-A_{r}\right) & R_{i}^{T}+P_{1} B_{i} & -P_{1} B_{r} \\ 0 & M_{i}^{2} & 0 & -R_{i}^{T} & 0 \\ * & * & \bar{M}_{i}^{3} & 0 & P_{3} B_{r} \\ * & * & * & -P_{4}-P_{4}^{T} & 0 \\ * & * & * & * & -\eta^{2} I_{n_{u}}\end{array}\right)$ 


$$
\left(\begin{array}{ccccccccccc}
M_{i}^{1} & 0 & P_{1}\left(A_{i}-A_{r}\right) & R_{i}^{T}+P_{1} B_{i} & -P_{1} B_{r} & P_{1} \mathcal{A} & P_{1} \mathcal{A} & P_{1} \mathcal{B} & 0 & 0 & 0 \\
* & M_{i}^{2} & 0 & -R_{i}^{T} & 0 & 0 & 0 & 0 & P_{2} \mathcal{A} & P_{2} \mathcal{A} & P_{2} \mathcal{B} \\
* & * & M^{3} & 0 & P_{3} B_{r} & 0 & 0 & 0 & 0 & 0 & 0 \\
* & * & * & M^{4} & 0 & 0 & 0 & 0 & 0 & 0 & 0 \\
* & * & * & * & -\eta^{2} I_{n_{u}} & 0 & 0 & 0 & 0 & 0 & 0 \\
* & * & * & * & * & -\lambda_{1}^{1} I_{n n_{x}} & 0 & 0 & 0 & 0 & 0 \\
* & * & * & * & * & * & -\lambda_{3}^{1} I_{n n_{x}} & 0 & 0 & 0 & 0 \\
* & * & * & * & * & * & * & -\lambda_{4}^{1} I_{n n_{u}} & 0 & 0 & 0 \\
* & * & * & * & * & * & * & * & -\lambda_{1}^{2} I_{n n_{x}} & 0 & 0 \\
* & * & * & * & * & * & * & * & * & -\lambda_{3}^{2} I_{n n_{x}} & 0 \\
* & * & * & * & * & * & * & * & * & * & -\lambda_{1}^{2} I_{n n_{u}}
\end{array}\right)
$$

with

$$
\begin{aligned}
& \bar{M}_{i}^{1}=A_{i}^{T} P_{1}+P_{1} A_{i}+Q \\
& M_{i}^{2}=P_{2} A_{i}+A_{i}^{T} P_{2}-C^{T} F_{i}^{T}-F_{i} C \\
& \bar{M}_{i}^{3}=A_{r}^{T} P_{3}+P_{3} A_{r} \\
& R_{i}=P_{4}^{T} K_{i} \\
& F_{i}=P_{2} L_{i}
\end{aligned}
$$

and

$$
Q(t)=\left(\begin{array}{ccccc}
P_{1} \Delta A(t) & 0 & P_{1} \Delta A(t) & P_{1} \Delta B(t) & 0 \\
P_{2} \Delta A(t) & 0 & P_{2} \Delta A(t) & P_{2} \Delta B(t) & 0 \\
0 & 0 & 0 & 0 & 0 \\
0 & 0 & 0 & 0 & 0 \\
0 & 0 & 0 & 0 & 0
\end{array}\right)
$$

Using the time-varying terms structure defined in (9) and (10), $Q(t)$ is written as follows:

$$
Q(t)=X_{A} \Sigma_{A}(t) Y_{A}^{T}+X_{B} \Sigma_{B}(t) Y_{B}^{T}
$$

with

$$
\begin{aligned}
X_{A}= & \left(\begin{array}{c}
P_{1} \mathcal{A} \\
P_{2} \mathcal{A} \\
0 \\
0 \\
0 \\
E_{A}
\end{array}\right), X_{B}=\left(\begin{array}{c}
P_{1} \mathcal{B} \\
P_{2} \mathcal{B} \\
0 \\
0 \\
0
\end{array}\right) \\
Y_{A}= & \left(\begin{array}{c}
E_{A}^{T} \\
0 \\
E_{A}^{T} \\
0 \\
0
\end{array}\right), Y_{B}=\left(\begin{array}{c}
0 \\
0 \\
0 \\
E_{B}^{T} \\
0
\end{array}\right)
\end{aligned}
$$

Lemma 1. [20] For any matrices $X, Y, \Sigma(t)$ with $\Sigma(t) \Sigma^{T}(t) \leq I$ and any positive scalar $\lambda$, it holds:

$$
X \Sigma(t) Y^{T}+Y \Sigma^{T}(t) X^{T} \leq \lambda^{-1} X^{T} X+\lambda Y^{T} Y
$$

Based on the property given in the lemma 1 , the term
$Q(t)+Q^{T}(t)$ can be bounded as follows:

$$
Q(t)+Q^{T}(t)<\left(\begin{array}{ccccc}
Q_{1} & 0 & 0 & 0 & 0 \\
0 & Q_{2} & 0 & 0 & 0 \\
0 & 0 & Q_{3} & 0 & 0 \\
0 & 0 & 0 & Q_{4} & 0 \\
0 & 0 & 0 & 0 & 0
\end{array}\right)
$$

with

$$
\begin{aligned}
Q_{1}= & \left(\lambda_{1}^{1}+\lambda_{1}^{2}\right) E_{A}^{T} E_{A}+ \\
& \left(\left(\lambda_{1}^{1}\right)^{-1}+\left(\lambda_{3}^{1}\right)^{-1}\right) P_{1} \mathcal{A A}^{T} P_{1}+\left(\lambda_{4}^{1}\right)^{-1} P_{1} \mathcal{B B}^{T} P_{1} \\
Q_{2}= & \left(\left(\lambda_{1}^{2}\right)^{-1}+\left(\lambda_{3}^{2}\right)^{-1}\right) P_{2} \mathcal{A A}^{T} P_{2}+\left(\lambda_{4}^{2}\right)^{-1} P_{2} \mathcal{B B}^{T} P_{2} \\
Q_{3}= & \left(\lambda_{3}^{1}+\lambda_{3}^{2}\right) E_{A}^{T} E_{A} \\
Q_{4}= & \left(\lambda_{4}^{1}+\lambda_{4}^{2}\right) E_{B}^{T} E_{B}
\end{aligned}
$$

Then, replacing (31) and (35) in (30) and applying a Schur complement, the condition (27) ensuring the $\mathcal{L}_{2}$ tracking performance with the state estimation simultaneously is implied by LMI (21), which ends the proof.

\section{Numerical SimUlation}

The proposed control law and observer design ensuring the model reference tracking is illustrated by the following academic example.

Let us consider the nonlinear T-S system (1) with $n=2$, and

$$
\begin{aligned}
& A_{1}=\left(\begin{array}{ccc}
-1 & 1 & 0 \\
-6 & -5 & -1 \\
3 & 0 & -1
\end{array}\right), A_{2}=\left(\begin{array}{ccc}
-1 & 1 & 0 \\
-3 & -5 & -1 \\
-1 & -1 & -2
\end{array}\right) \\
& B_{1}=\left(\begin{array}{cc}
0 & 0 \\
0.4 & 0.1 \\
0 & 0.2
\end{array}\right), B_{2}=\left(\begin{array}{cc}
0 & 0 \\
-0.2 & -1 \\
1 & 0.5
\end{array}\right) \\
& C=\left(\begin{array}{lll}
1 & 0 & 0
\end{array}\right)
\end{aligned}
$$

The weighting functions depend on the system states and are defined as follows

$$
\begin{aligned}
& h_{1}(x(t))=\frac{2-\sin \left(x_{1}(t)\right)-\tanh \left(x_{2}(t)\right)}{4} \\
& h_{2}(x(t))=1-h_{1}(x(t))
\end{aligned}
$$

The reference model (3) is defined by the following matrices:

$$
A_{r}=\left(\begin{array}{ccc}
-1 & 1 & 0 \\
-2 & -8 & -1 \\
-1 & -2 & -5
\end{array}\right), B_{r}=\left(\begin{array}{ll}
0 & 0 \\
1 & 1 \\
1 & 2
\end{array}\right)
$$


and the input $u_{r}(t)$ which is known and bounded (see figure 3).

The observer and controller are respectively defined by equations (6) and (16), their gains are calculated by solving the LMI problem given by (21). The obtained results are :

$$
\begin{aligned}
& K_{1}=\left(\begin{array}{ccc}
20.6280 & -381.3725 & -47.2350 \\
10.0786 & -90.8162 & -137.3001
\end{array}\right) \\
& K_{2}=\left(\begin{array}{ccc}
13.4233 & -320.8239 & -858.9901 \\
-56.9571 & 863.5566 & 66.2848
\end{array}\right) \\
& L_{1}=\left(\begin{array}{lll}
-0.6914 & -3.2299 & 3.1567
\end{array}\right)^{T} \\
& L_{2}=\left(\begin{array}{lll}
-0.6954 & -0.1777 & -0.8158
\end{array}\right)^{T}
\end{aligned}
$$

Figures 1 and 2 depict the system and observer states (respectively denoted $x$ and $\hat{x}$ ), and the system and reference states (respectively denoted $x$ and $x_{r}$ ). Figure 3 depicts the reference input and the tracking control (respectively denoted $u_{r}$ and $u$ ). The weighting functions $h_{i}(x)(i=1,2)$ and $h_{i}(\hat{x})(i=1,2)$ are depicted in figure 4.
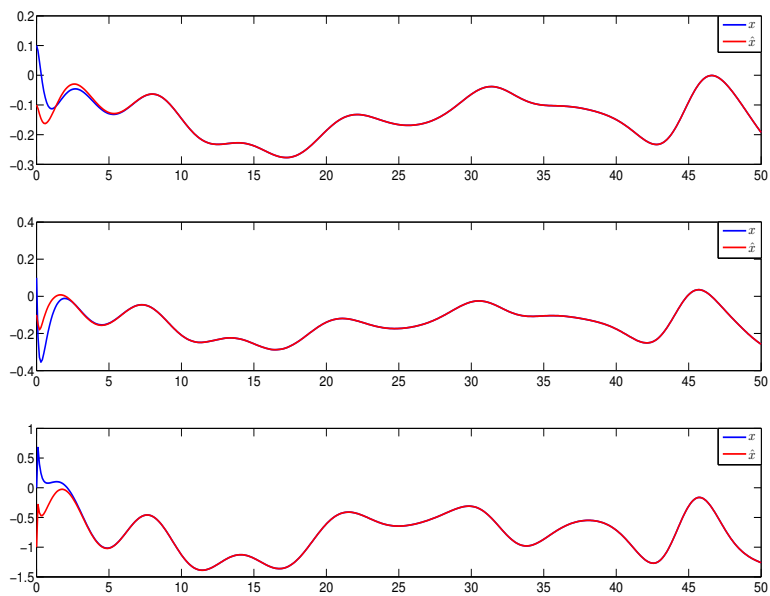

Fig. 1. System and observer states

Figure 1 shows the tracking state trajectory and its estimate with initial condition $x(0)=\left(\begin{array}{lll}0.1 & 0.1 & 0\end{array}\right)^{T}$, observer state $\hat{x}(0)=\left(\begin{array}{lll}-0.1 & -0.1 & -1\end{array}\right)^{T}$ and the model reference state $x_{r}(0)=\left(\begin{array}{lll}0.2 & 0 & 0\end{array}\right)^{T}$. The tracking performances were fixed by choosing $Q=10^{-3} I_{3}$ and optimizing the attenuation level $\eta^{2}$.

The depicted figures show that the state trajectory follow the model reference with an efficient estimation of the system state despite the fact that the weighting functions have unmeasurable premise variables.

\section{THE MEASUREMENT NOISE CASE}

In order to improve the efficiency of the proposed approach, we consider the presence of measurement noise. The system
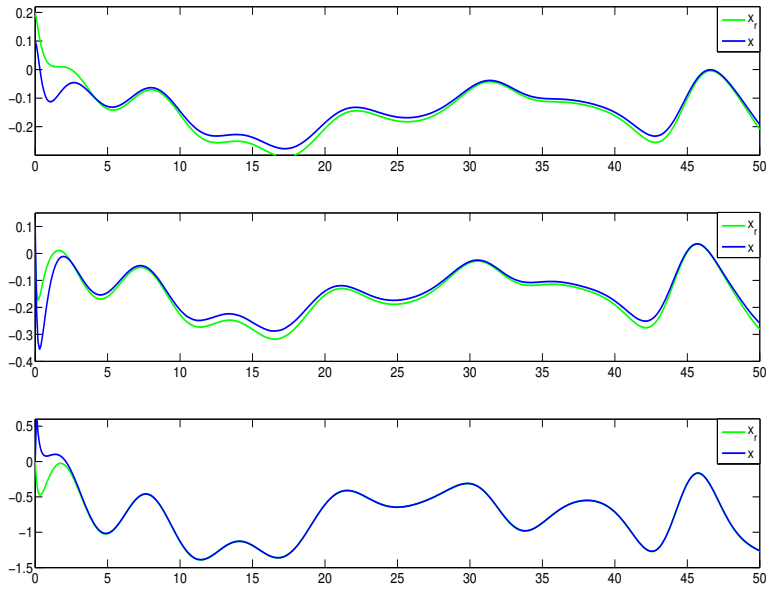

Fig. 2. System and model reference states
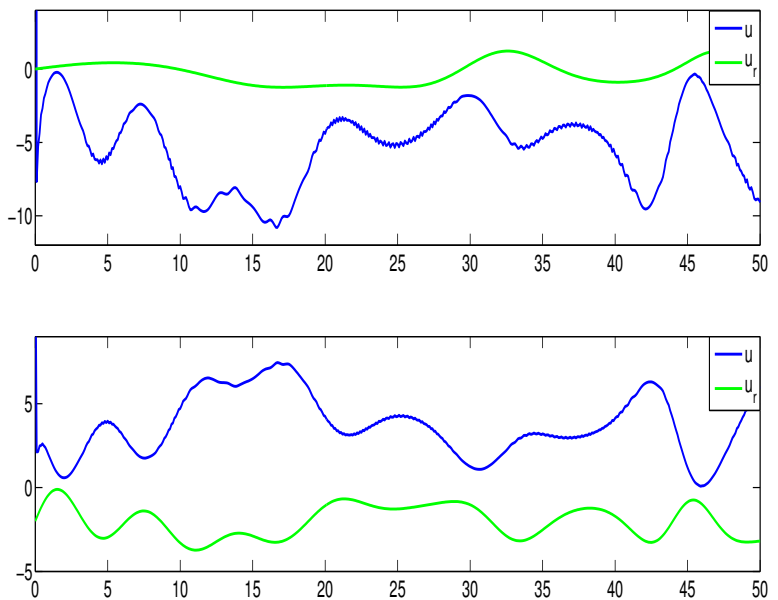

Fig. 3. Model reference and System inputs
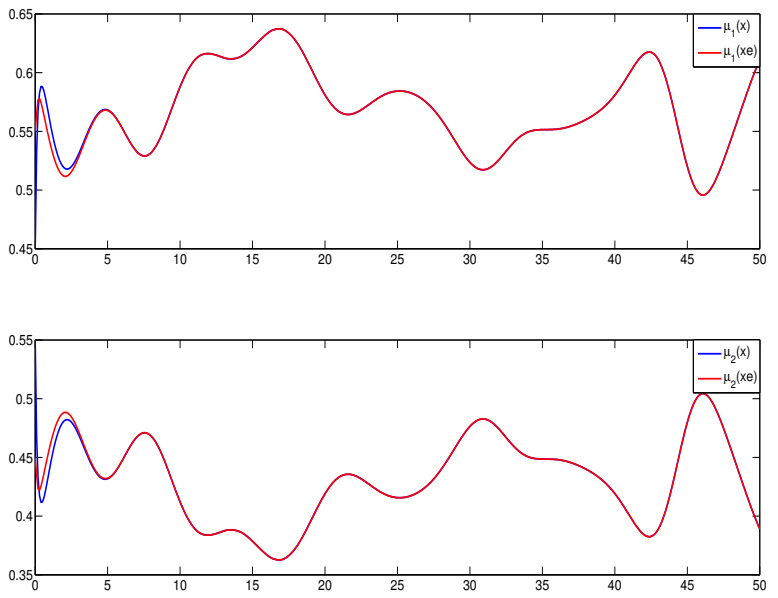

Fig. 4. The weighting functions $\mu_{i}(x)(i=1,2)$ and $\mu_{i}(\hat{x})(i=1,2)$ 
is then described by the following equations:

$$
\left\{\begin{array}{l}
\dot{x}(t)=\sum_{i=1}^{n} h_{i}(x(t))\left(A_{i} x(t)+B_{i} u(t)\right) \\
y(t)=C x(t)+G b(t)
\end{array}\right.
$$

where $b(t)$ is the noise measurement and matrices $A_{i}, B_{i}$ and $C$ have already been defined in (1).

The model reference and state observer are defined as in equations (3) and (6).

Let us consider the augmented vectors $x_{a}(t)$

$$
x_{a}(t)=\left(\begin{array}{llll}
e_{r}^{T}(t) & e_{x}^{T}(t) & x_{r}^{T}(t) & u^{T}(t)
\end{array}\right)^{T}
$$

and $u_{a}(t)$ now takes into account the noise $b(t)$

$$
u_{a}(t)=\left(\begin{array}{ll}
u_{r}^{T}(t) & b^{T}(t)
\end{array}\right)^{T}
$$

Using the descriptor approach of section III, It follows:

$$
E \dot{x}_{a}(t)=\sum_{i=1}^{n} h_{i}(\hat{x}(t))\left(\bar{A}_{i}(t) x_{a}(t)+\bar{B}_{i} u_{a}(t)\right)
$$

with

$$
\begin{aligned}
& \bar{A}_{i}(t)=\left(\begin{array}{cccc}
A_{i}+\Delta A(t) & 0 & A_{i}-A_{r}+\Delta A(t) B_{i}+\Delta B(t) \\
\Delta A(t) & A_{i}-L_{i} C & \Delta A(t) & \Delta B(t) \\
0 & 0 & A_{r} & 0 \\
K_{i} & -K_{i} & 0 & -I_{n_{u}}
\end{array}\right) \\
& E=\left(\begin{array}{cccc}
I_{n_{x}} & 0 & 0 & 0 \\
0 & I_{n_{x}} & 0 & 0 \\
0 & 0 & I_{n_{x}} & 0 \\
0 & 0 & 0 & 0
\end{array}\right), \bar{B}_{i}=\left(\begin{array}{cc}
-B_{r} & 0 \\
0 & -L_{i} G \\
B_{r} & 0 \\
0 & 0
\end{array}\right)
\end{aligned}
$$

The objective is to attenuate the effect of noise on the state estimation and the tracking such that the system state is ensured to be as close as possible to the reference state. The computation of the observer and controller gains is detailed in the next theorem

Theorem 2. There exist a PDC state feedback controller (16) and an observer (6) for a nonlinear system described by a $T$ $S$ model (40) and subject to noise measurement guaranteeing the $\mathcal{L}_{2}$ tracking performance (5) with a prescripted value $\eta^{2}$, if there exists matrices $P_{1}=P_{1}^{T}>0, P_{2}=P_{2}^{T}>0, P_{3}=$ $P_{3}^{T}>0, P_{4}>0, F_{i}, R_{i}$, positive scalars $\lambda_{1}$ and $\lambda_{2}$ satisfying the linear matrix inequalities (21) for $i=1, \ldots, n$. with

$$
\begin{aligned}
& M_{i}^{1}=A_{i}^{T} P_{1}+P_{1} A_{i}+Q+\left(\lambda_{1}^{1}+\lambda_{1}^{2}\right) E_{A}^{T} E_{A} \\
& M_{i}^{2}=P_{2} A_{i}+A_{i}^{T} P_{2}-C^{T} F_{i}^{T}-F_{i} C \\
& M^{3}=A_{r}^{T} P_{3}+P_{3} A_{r}+\left(\lambda_{3}^{1}+\lambda_{3}^{2}\right) E_{A}^{T} E_{A} \\
& M^{4}=-P_{4}-P_{4}^{T}+\left(\lambda_{4}^{1}+\lambda_{4}^{2}\right) E_{B}^{T} E_{B}
\end{aligned}
$$

The controller and observer gains are given $(i=1, \ldots, n)$ by

$$
\begin{aligned}
& K_{i}=\left(P_{4}^{T}\right)^{-1} R_{i} \\
& L_{i}=P_{2}^{-1} F_{i}
\end{aligned}
$$

Remark 2. Same remark as in the previous section can be made concerning the optimization of the attenuation value $\eta^{2}$.

Proof: The proof of Theorem 2 is based on the same development as the previous proof and is thus omitted.

\section{Numerical Simulation}

Let us consider the same numerical example as in section IV with a measurement noise defined by a normal distribution with zero mean and standard deviation $20 \%$ of the output magnitude affecting the system (36) with $G=1$.

Applying theorem 2, the observer and controller gains are given by:

$$
\begin{aligned}
& K_{1}=\left(\begin{array}{ccc}
6.5964 & -661.8742 & -153.4759 \\
50.6975 & -183.0979 & -236.4476
\end{array}\right) \\
& K_{2}=\left(\begin{array}{ccc}
-31.6748 & -61.0740 & -1338.78 \\
-41.4662 & 1519.11 & 214.5846
\end{array}\right) \\
& L_{1}=\left(\begin{array}{lll}
-0.3159 & -0.9728 & 1.2097
\end{array}\right)^{T} \\
& L_{2}=\left(\begin{array}{lll}
-0.3331 & 0.2301 & -1.0032
\end{array}\right)^{T}
\end{aligned}
$$

In order to show the efficiency of the proposed noise attenuation, the gains of the observer (6) and of the controller (16) where first computed without taking into account the noise (applying theorem 1) and the obtained results are displayed on figure 5. The state trajectories obtained with the same inputs, but using the observer/controller computed from theorem 2 allowing the noise attenuation, are displayed on figure 6. One can see the better matching of $\hat{x}(t)$ and $x(t)$ to $x_{r}(t)$ in the second case.

From the depicted figures, one can see that, if the noise is not taken into account, it affects and even degrades the model reference tracking. Whereas, when it is included in the controller and observer synthesis, even if the measurements are affected by noises, acceptable state tracking and estimation are obtained.

For the considered example, in order to quantify the obtained improvement for the tracking error, the following criterion is considered. Let us denote $\Phi_{i}=\sum_{t}\left(x_{r i}(t)-\hat{x}_{i}(t)\right)$ for the state estimate obtained from theorem 2 (results depicted in figure 6) and $\bar{\Phi}_{i}=\sum_{t}\left(x_{r i}(t)-\hat{x}_{i}(t)\right)$ for the results obtained without taking into account the noise in the development (results depicted in figure 5).

The comparative criterion $\tau_{i}$ for each state is defined as:

$$
\tau_{i}=\frac{\bar{\Phi}_{i}-\Phi_{i}}{\bar{\Phi}_{i}} 100 \%
$$

and is equal to $\tau_{1}=33.97 \%, \tau_{2}=58.05 \%$ and $\tau_{3}=28.62 \%$ respectively for each state.

\section{CONCLUSION}

In the present paper, the problem of model reference tracking of nonlinear systems represented by a T-S structure has been considered. Based on a Parallel Distributed Compensation state feedback law, the tracking error is reduced in terms of an $\mathcal{L}_{2}$ gain attenuation. The contribution of the presented approach is that it is based on the descriptor strategy, avoiding the crossing terms between the feedback gains and the Lyapunov matrices and reducing the number of LMI to solve. As a consequence, relaxed LMI conditions are obtained.

The second point is about the state observer synthesis. One 


$\left(\begin{array}{cccccccccccc}M_{i}^{1} & 0 & P_{1}\left(A_{i}-A_{r}\right) & R_{i}^{T}+P_{1} B_{i} & -P_{1} B_{r} & 0 & P_{1} \mathcal{A} & P_{1} \mathcal{A} & P_{1} \mathcal{B} & 0 & 0 & 0 \\ * & M_{i}^{2} & 0 & -R_{i}^{T} & 0 & -F_{i} G & 0 & 0 & 0 & P_{2} \mathcal{A} & P_{2} \mathcal{A} & P_{2} \mathcal{B} \\ * & * & M^{3} & 0 & P_{3} B_{r} & 0 & 0 & 0 & 0 & 0 & 0 & 0 \\ * & * & * & M^{4} & 0 & 0 & 0 & 0 & 0 & 0 & 0 & 0 \\ * & * & * & * & -\eta^{2} I_{n_{u}} & 0 & 0 & 0 & 0 & 0 & 0 & 0 \\ * & * & * & * & * & -\eta^{2} I_{n_{y}} & 0 & 0 & 0 & 0 & 0 & 0 \\ * & * & * & * & * & * & -\lambda_{1}^{1} I_{n n_{x}} & 0 & 0 & 0 & 0 & 0 \\ * & * & * & * & * & * & * & -\lambda_{3}^{1} I_{n n_{x}} & 0 & 0 & 0 & 0 \\ * & * & * & * & * & * & * & * & -\lambda_{4}^{1} I_{n n_{u}} & 0 & 0 & 0 \\ * & * & * & * & * & * & * & * & * & -\lambda_{1}^{2} I_{n n_{x}} & 0 & 0 \\ * & * & * & * & * & * & * & * & * & * & -\lambda_{3}^{2} I_{n n_{x}} & 0 \\ * & * & * & * & * & * & * & * & * & * & * & -\lambda_{1}^{2} I_{n n_{n}} \\ & * & & & * & & * & & & * & & *\end{array}\right.$
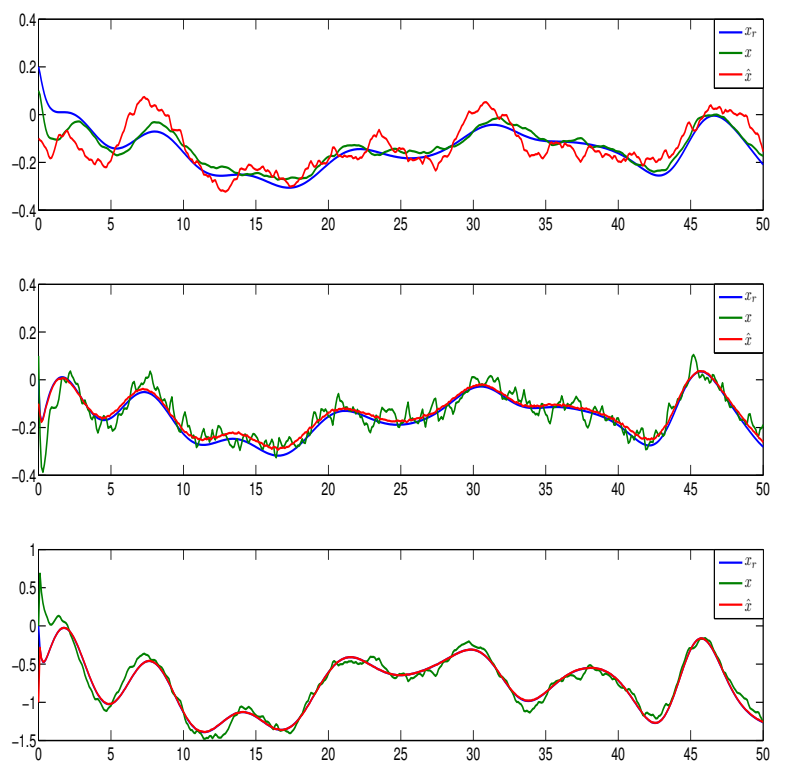

Fig. 5. System, observer and model reference states (theorem 1 with noise)

can note that the general case of unmeasurable premise variable depending on the system state is considered here for observer/controller design.

The simultaneous observation and control problem have been formulated in terms of LMI and a numerical example with convincing results was presented to illustrate the efficiency of the proposed method even in the presence of measurement noise.

\section{REFERENCES}

[1] B. Chen, H. Uang, and C. Tseng, "Robust tracking enhancement of robot systems including motor dynamics : a fuzzy-based dynamic game approach," IEEE Transactions on Fuzzy Systems, vol. 6, no. 4, pp. 538$552,1998$.

[2] W. Wang and H. Lin, "Fuzzy control design for the trajectory tracking
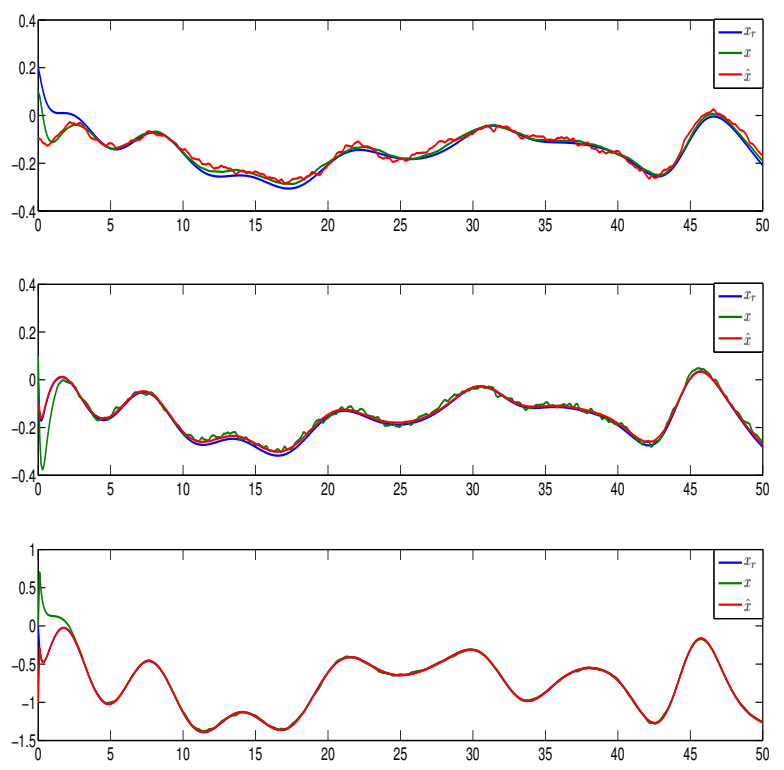

Fig. 6. System, observer and model reference states (theorem 2 with noise)

on uncertain nonlinear system," IEEE Transactions on Fuzzy Systems, vol. 7, no. 1, pp. 53-62, 1999.

[3] T. Takagi and M. Sugeno, "Fuzzy identification of systems and its applications to modeling and control," IEEE Transactions on Systems, Man, and Cybernetics, vol. 15, no. 1, pp. 116-132, 1985.

[4] A. Akhenak, M. Chadli, J. Ragot, and D. Maquin, "Design of sliding mode unknown input observer for uncertain Takagi-Sugeno model," in In $15^{\text {th }}$ Mediterranean Conference on Control and Automation, MED07, Athens, Greece, 2007.

[5] B. Marx, D. Koenig, and J. Ragot, "Design of observers for TakagiSugeno descriptor systems with unknown inputs and application to fault diagnosis," IET Control Theory and Applications, vol. 1, no. 5, pp. 1487-1495, 2007.

[6] K. Tanaka and H. Wang, Fuzzy Control Systems Design and Analysis: A Linear Matrix Inequality Approach. John Wiley \& Sons, 2001.

[7] T. Taniguchi, K. Tanaka, K. Yumafuji, and H. Wang, "A new PDC fuzzy reference models," in IEEE International Fuzzy Systems Conference, Seoul, South Korea, 1999. 
[8] C. Tseng and B. Chen, " $H_{\infty}$ decentralized fuzzy model reference tracking control design for nonlinear interconnected systems," IEEE Transactions on Fuzzy Systems, vol. 9, no. 6, pp. 795-809, 2001.

[9] B. Mansouri, N. Manamanni, K. Guelton, A. Kruszewski, and T. Guerra, "Output feedback LMI tracking control conditions with $H_{\infty}$ criterion for uncertain and disturbed T-S models," Information Sciences, vol. 179, pp. 446-457, 2009.

[10] C.-S. Tseng, B.-S. Chen, and H. Uang, "Fuzzy tracking control design for nonlinear dynamic systems via T-S fiuzzy model," IEEE Transactions on Fuzzy Systems, vol. 9, no. 3, pp. 381-392, 2001.

[11] B. Chen, C. Lee, and Y. Chang, " $H_{\infty}$ tracking design for uncertain nonlinear SISO systems: Adaptive fuzzy approach,” IEEE Transactions on Fuzzy Systems, vol. 4, no. 1, pp. 32-43, 1996.

[12] S. Tong, T. Wang, and H.-X. Li, "Fuzzy robust tracking control for uncertain nonlinear systems," International Journal of Approximate Reasoning, vol. 30, no. 2, pp. 73-90, 2002.

[13] D. Ichalal, B. Marx, J. Ragot, and D. Maquin, "Advances in observer design for Takagi-Sugeno systems with unmeasurable premise variables," in $20^{\text {th }}$ Mediterranean Conference on Control and Automation, MED12, Barcelona, Spain, 2012.

[14] K. Tanaka and H. Wang, Fuzzy Control Systems Design and Analysis: A Linear Matrix Inequality Approach. John Wiley \& Sons, 2001.

[15] D. Luenberger, "Dynamic equation in descriptor form," IEEE Transactions on Automatic Control, vol. 22, no. 3, pp. 312-321, 1977.

[16] K. Guelton, T. Bouarar, and N. Manamanni, "Robust dynamic output feedback fuzzy Lyapunov stabilization of Takagi-Sugeno systems- a descriptor redundancy approach," Fuzzy Sets and Systems, vol. 160, no. 19, pp. 2796-2811, 2009.

[17] B. Marx and J. Ragot, "Stability and $L_{2}$ norm bound conditions for Takagi-Sugeno descriptor systems," in IFAC World Congress, Seoul, South Korea, 2008.

[18] B. Marx, D. Koenig, and D. Georges, "Robust fault tolerant control for descriptor systems," IEEE Transactions on Automatic Control, vol. 49, pp. 1869-1875, 2004.

[19] K. Tanaka, H. Ohtake, and H. Wang, "A descriptor system approach to fuzzy control system design via fuzzy Lyapunov functions," IEEE Transactions on Fuzzy Systems, vol. 15, no. 3, pp. 333-341, 2007.

[20] K. Zhou and P. Khargonekar, "Robust stabilization of linear systems with norm-bounded time-varying uncertainty," Systems and Control Letters, vol. 10, no. 1, pp. 17-20, 1988. 